\title{
Ablation and Wind Mass-Loading in the Born-Again Planetary Nebula A 30
}

\author{
Martín A. Guerrero ${ }^{1}$, You-Hua $\mathrm{Chu}^{2}$, Wolf-Rainer Hamann ${ }^{3}$, Lidia \\ Oskinova $^{3}$, Detlef Schönberner ${ }^{4}$, Helge Todt ${ }^{3}$, Matthias Steffen ${ }^{4}$, \\ Nieves Ruiz ${ }^{1}$, Robert A. Gruendl ${ }^{2}$ and William P. Blair ${ }^{5}$ \\ ${ }^{1}$ Instituto de Astrofísica de Andalucía (IAA-CSIC), Granada, Spain \\ email: mar@iaa.es, nieves@iaa.es \\ ${ }^{2}$ Astronomy Department, University of Illinois at Urbana-Champaign (UIUC), Urbana, USA \\ email: yhchu@astro.illinois.edu, gruendl@astro.illinois.edu \\ ${ }^{3}$ Institute for Physics and Astronomy, Universität Potsdam, Potsdam, Germany \\ email: wrh@astro.physik.uni-potsdam.de, lida@astro.physik.uni-potsdam.de, \\ htodt@astro.physik . uni-potsdam.de \\ ${ }^{4}$ Leibniz-Institut für Astrophysik Potsdam (AIP), Potsdam, Germany \\ email: deschoenberner@aip.de, msteffen@aip.de \\ ${ }^{5}$ Department of Physics and Astronomy, Johns Hopkins University (PHA-JHU), Baltimore, \\ USA \\ email: wpb@pha.jhu.edu
}

\begin{abstract}
We present $X M M-N e w t o n$ and Chandra observations of the born-again planetary nebula A 30. These X-ray observations reveal a bright unresolved source at the position of the central star whose X-ray luminosity exceeds by far the model expectations for photospheric emission and for shocks within the stellar wind. We suggest that a "born-again hot bubble" may be responsible for this X-ray emission. Diffuse X-ray emission associated with the petal-like features and one of the H-poor knots seen in the optical is also found. The weakened emission of carbon lines in the spectrum of the diffuse emission can be interpreted as the dilution of stellar wind by mass-loading or as the detection of material ejected during a very late thermal pulse.
\end{abstract}

Keywords. planetary nebulae: individual (A 30), X-rays: ISM

The planetary nebula (PN) A 30 is one of the few born-again PNe, where thermonuclear burning of $\mathrm{H}$ in the remnant stellar envelope built up He until it ignited its fusion into $\mathrm{C}$ and O during a late thermal pulse. At optical wavelengths (e.g., Borkowski et al. 1995; Chu et al. 1997), A 30 consists of (a) an old, round shell with $2^{\prime}$ in size, (b) an intricate system of arcs or petal-like features, best seen in [O III], (c) a disk and two bipolar outflows of H-poor knots, and (d) a H-deficient [WC]-PG1159 type central star (CSPN).

ROSAT PSPC observations of A 30 revealed a source of soft X-ray emission from a plasma at a temperature $\sim 3 \times 10^{5} \mathrm{~K}$ (Chu \& Ho 1995). Follow-up HRI observations showed a central point source and hints of diffuse emission (Chu et al. 1997). We have obtained new X-ray observations of A 30 using XMM-Newton (PI: Hamann) and Chandra (PI: Chu) with net exposure times $\sim 31 \mathrm{ks}$ for XMM-Newton EPIC-MOS and RGS, $\sim 25 \mathrm{ks}$ for XMM-Newton EPIC-pn, and $96.1 \mathrm{ks}$ for Chandra ACIS-S.

$X M M$-Newton detects a bright, soft $(<0.6 \mathrm{keV})$ source at the CSPN with an EPIC-pn count rate in the $0.22-0.6 \mathrm{keV}$ energy band of $39.6 \pm 1.3$ counts ks ${ }^{-1}$. Chandra also detects this point source with a count rate of $1.80 \pm 0.14$ counts $\mathrm{ks}^{-1}$ in the same energy band. In addition, XMM-Newton confirms the presence of faint extended emission at distances $\geqslant 13^{\prime \prime}$ that fills the [O III] petal-like features, while Chandra suggests tantalizing evidence of emission associated with the southwest cometary knots. 

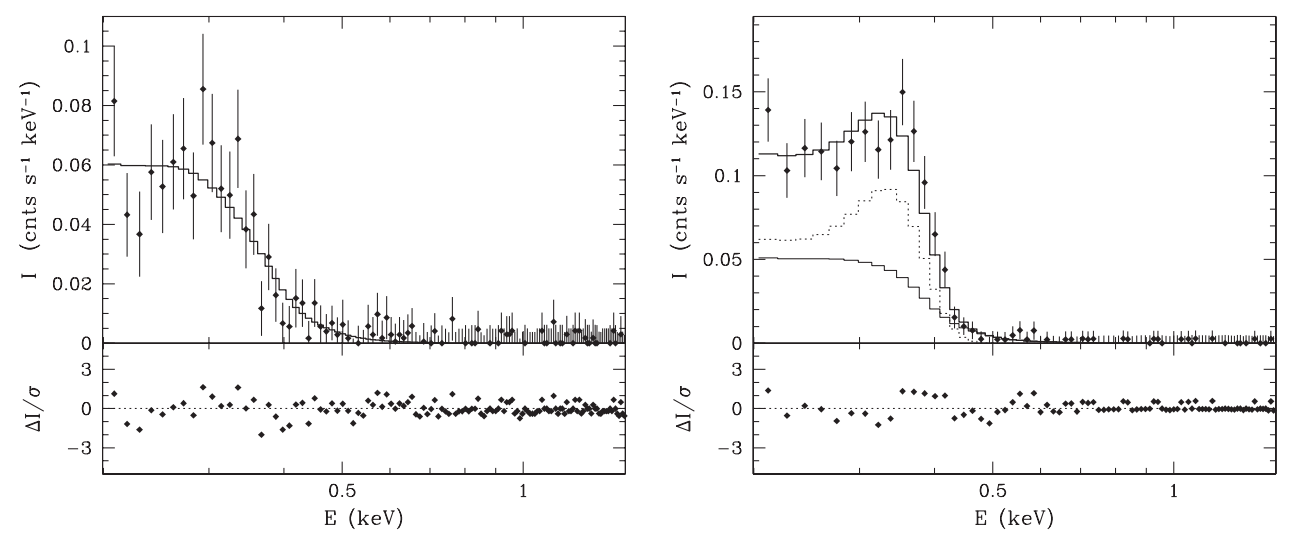

Figure 1. XMM-Newton background-subtracted spectra overplotted with best-fit models of the diffuse (left) and stellar (right) emissions. The latter shows the C VI emission line (dotted histogram) added to the thermal component with Leuenhagen et al.'s (1993) chemical abundances (thin histogram) that suggests higher carbon abundances than the diffuse emission.

The spectrum of the diffuse emission (Figure 1-left) has been modeled using an absorbed optically thin plasma emission model, adopting Leuenhagen et al.'s (1993) chemical abundances and a foreground hydrogen column density $\left(N_{\mathrm{H}}\right)$ of $5 \times 10^{20} \mathrm{~cm}^{-2}(\mathrm{Chu}$ \& Ho 1995) with best-fit parameters $T_{\mathrm{X}} \approx 7.9 \times 10^{5} \mathrm{~K}$ and $L_{\mathrm{X}} \approx 6.0 \times 10^{31} \mathrm{erg} \mathrm{s}^{-1}(\mathrm{~d}=$ $2.4 \mathrm{kpc}$ ). This model, however, does not provide a good fit to the spectrum of the CSPN (Figure 1-right) that shows emission excess at $\sim 0.36 \mathrm{keV}$. The addition of an emission line, suggested by the RGS spectra to be the $\mathrm{C}$ VI $L_{\alpha}$ line at $33.7 \AA$, matches better the CSPN spectrum with best-fit parameters $T_{\mathrm{X}} \approx 9.4 \times 10^{5} \mathrm{~K}$ and $L_{\mathrm{X}} \approx 1.2 \times 10^{32} \mathrm{erg} \mathrm{s}^{-1}$.

The higher $\mathrm{C}$ abundances of the CSPN with respect to the diffuse emission may imply in the latter the dilution of the stellar wind by mass-loading, or the detection of emission from material ejected during a late thermal pulse. As for the CSPN, we may consider:

- Photospheric emission of the hot CSPN: The expected X-ray flux is $10^{10}$ times lower than observed, as radiation is blocked by CNO bound-free and K-shell opacities.

- Shocks within the stellar winds: Shocks embedded in the radiation driven winds of hot stars can produce X-ray emission (e.g., Feldmeier et al. 1997). For the canonical $L_{\mathrm{X}} / L_{\mathrm{bol}} \approx 10^{-7}$ relationship of O stars (Berghoefer et al. 1997), A 30 CSPN would be 100 times fainter than observed. Moreover, this [WC]-PG1159 CSPN is expected to be $\mathrm{X}$-ray fainter than $\mathrm{O}$ stars, because the winds of WC stars are very opaque to the X-rays produced in zones of strong wind acceleration at few stellar radii (Oskinova et al. 2003).

- "Born-again" hot bubble: The CSPN wind mechanical luminosity, $\sim 10^{35} \mathrm{erg} \mathrm{s}^{-1}$, is $\sim 800$ times larger than the observed $L_{\mathrm{X}}$, and its spectrum is consistent with thermal emission. Thus, our favored interpretation is that X-rays originate from hot plasma generated by the present fast stellar wind ramming into previously ejected slow material.

\section{References}

Berghoefer, T. W., Schmitt, J. H. M. M., Danner, R., \& Cassinelli, J. P. 1997, A\& A, 322, 167

Borkowski, K. J., Harrington, J. P., \& Tsvetanov, Z. I. 1995, ApJ, 449, L143

Chu, Y.-H. \& Ho, C.-H. 1995, ApJ, 448, L127

Chu, Y.-H., Chang, T. H., \& Conway, G. M. 1997, ApJ, 482, 891

Feldmeier, A., Puls, J., \& Pauldrach, A. W. A. 1997, A\& A, 322, 878

Leuenhagen, U., Koesterke, L., \& Hamann, W.-R. 1993, AcA, 43, 329

Oskinova, L. M., Ignace, R., Hamann, W.-R., Pollock, A. M. T., \& Brown, J. C. 2003, A\&A, 402,755 